\title{
PREDIÇÃO DO DESEMPENHO A PARTIR DAS \\ CARACTERÍSTICAS ANTROPOMÉTRICAS, \\ FISIOLÓGICAS E DE FORÇA NO REMO
}

Artigo Original

ORIGINAL ARTICLE

PREDICTION OF PERFORMANCE FROM ANTHROPOMETRIC, PHYSIOLOGICAL ANDSTRENGTH

Artículo Original

COMPONENTS INROWING

PREDICCIÓN DEL RENDIMIENTO DE LAS CARACTERÍSTICAS ANTROPOMÉTRICAS, FISIOLÓGICAS

Y FUERZA EN EL REMO

Fábio Barreto Maia da Silva' (Profissional de Educação Física)

João Paulo Reis Gonçalves Moreira de Brito ${ }^{2}$

(Profissional de Educação Física)

Victor Machado Reis ${ }^{3}$

(Profissional de Educação Física)

1. Escola Naval, Niterói, Rio de

Janeiro, RJ, Brasil.

2. Instituto Politécnico de Santarém,

Escola Superior de Desporto

de Rio Maior, Rio Maior,

Santarém, Portugal.

3. Centro de Investigação

em Desporto, Saúde e

Desenvolvimento Humano,

CIDESD, Vila Real, Portugal.

\section{Correspondência:}

Rua Visconde de Sepetiba, 50, apto. 803, Niterói, Rio de Janeiro, RJ, Brasil. 24020-206.fbms78@hotmail.com

\section{RESUMO}

Introdução: O desempenho de remadores no remoergômetro é motivo de curiosidade entre os cientistas. Os modelos de predição podem medir o desempenho no remoergômetro. Neste sentido, as variáveis estudadas foram analisadas como possíveis preditores de desempenho. Objetivo: Desenvolver diferentes modelos de regressão a fim de predizer o desempenho com o uso das variáveis antropométricas composição corporal, consumo máximo de oxigênio e força. Métodos: Vinte remadores participaram do estudo (21,35 $\pm 0,98$ anos). Foram aplicados os testes de consumo máximo de oxigênio, força, 500 m e o exame de absorciometria radiológica de dupla energia. A regressão linear múltipla foi realizada no SPSS 16 para os quatro diferentes modelos de regressão. A confiabilidade dos modelos foi indicada pelo coeficiente de determinação R2 e pelo erro padrão da estimativa (SEE). Resultados: Os modelos de antropometria-potência ( $R 2=0,92 ; \mathrm{SEE}=0,06), \mathrm{VO}_{2} \mathrm{Pico}(\mathrm{R} 2=0,88 ; \mathrm{SEE}=0,07)$, força-potência ( $\mathrm{R} 2=0,93 ; \mathrm{SEE}=0,06$ ) apresentaram predição confiável para o desempenho nos $500 \mathrm{~m}$ em remoergômetro, assim como a combinação de todas as variáveis ( $R 2=0,94 ; \mathrm{SEE}=0,08$ ). Conclusão: Feitas essas análises, pode-se assegurar a necessidade desses modelos com o objetivo de complementar a identificação, seleção de talentos e, sobretudo, melhora no desempenho.

Descritores: desempenho atlético; força muscular; antropometria; análise de variância; análise de regressão.

\section{ABSTRACT}

Introduction: The performance of rowers in the rowing ergometer is a matter of curiosity among scientists. Prediction models can measure performance on the rowing ergometer. In this sense, the studied variables were analyzed as possible predictors of performance. Objective: To develop different regression models in order to predict performance using the anthropometric variables body composition, maximal oxygen consumption, and strength. Methods: Twenty rowers participated in the study (21.35 \pm 0.98 years). The tests of maximal oxygen consumption, strength, $500 \mathrm{~m}$, and dual-energy $X$-ray absorptiometry were applied. Multiple linear regressions were performed on SPSS 16 for the four different regression models. The reliability of the models was indicated by the coefficient of determination $R 2$ and by the standard error of the estimation (SEE). Results: The anthropometry-power models ( $R 2=0.92$, SEE $=0.06)$, VO2 peak ( $R 2=0.88, S E E=0.07)$, strength-power ( $R 2=0.93$, SEE $=0.06$ ) presented reliable prediction for $500 \mathrm{~m}$ in the rowing ergometer, as well as the combination of all the variables $(R 2=0.94, S E E=0.08)$. Conclusion: Once these analyzes have been made, we can assurethenecessity of these models with the objective of complementing the identification, selection of talents and, above all, improvement in performance.

Keywords: athletic performance; muscle strength; anthropometry; analysis of variance; regression analysis.

\section{RESUMEN}

Introducción: El rendimiento de los remeros en el remoergómetro es motivo de curiosidad entre los científicos. Los modelos de predicción pueden medir el rendimiento en el remoergómetro. En este sentido, las variables estudiadas fueron analizadas como posibles predictores de rendimiento. Objetivo: Desarrollar diferentes modelos de regresión a fin de predecir el rendimiento con el uso de las variables antropométricas, composición corporal, consumo máximo de oxígeno y fuerza. Métodos: Veinte remeros participaron del estudio (21,35 $\pm 0,98$ años). Se aplicaron las pruebas de consumo de máximo oxígeno, fuerza, 500 m y el examen de absorciometría de rayos $X$ de doble energía. La regresión lineal múltiple fue realizada en el SPSS 16 para los cuatro diferentes modelos de regresión. La confiabilidad de los modelos fue indicada por el coeficiente de determinación R2 y el error estándar de la estimación (SEE). Resultados: Los modelos de antropometría-potencia $(R 2=0,92 ;$ SEE $=0,06), V \mathrm{~V}_{2}$ pico $(R 2=0,88 ;$ SEE $=0,07)$, fuerza-potencia $(R 2=0,93 ;$ SEE = 0,06) presentaron predicción confiable para el rendimiento en los 500 m en remoergómetro, así como la combinación de todas las variables $(R 2=0,94 ; S E E=0,08)$. Conclusión: Hechos estos análisis, se puede asegurar la necesidad de esos modelos con el objetivo de complementar la identificación, selección de talentos y, sobre todo, mejora en el rendimiento.

Descriptores: rendimiento atlético; fuerza muscular; antropometría; análisis de varianza; análisis de regresión. 


\section{INTRODUÇÃO}

O Remo em escaler (RE) é uma embarcação que permite 10 remadores mais o timoneiro. Na competição, o barco escaler possui um peso mínimo de 900 kg e de percorrer uma demarcação no mar, lagoa ou rio, chamado de raia, numa distância de 500 metros no menor tempo possível. As provas duram de dois minutos 40 segundo a três minutos, conforme dados da Federação de Remo do Estado do Rio de Janeiro (FRERJ). Esse tempo de prova demonstra grande ênfase no sistema anaeróbico. Akça ${ }^{1}$ acrescentou que grande contribuição e eficiência de energia anaeróbica pode ser preditor significativo de desempenho no remo.

Por causa de condições ambientais adversas e dificuldade de controlar o ambiente na raia de treinamento e competição, o remoergômetro vem sendo utilizado por preparadores e técnicos, como estratégia de manter e aumentar o condicionamento físico² ${ }^{2}$ e ainda possibilita a avaliação precisa das respostas metabólicas e cardiorrespiratórias durante o exercício ${ }^{3-5}$ bem como da performance ${ }^{3,6,7}$. Com a avaliação individual do remador, os técnicos são capazes de avaliar o ranking de cada atleta na equipe em ambiente controlado'.

Assim, os resultados das avaliações de potência e força máxima são preditores de desempenho no remoergômetro no teste máximo de 2000 metros $^{8-11}$. Além disso, o comportamento físico está relacionado com a composição e dimensão corporal do remador de alto nível, por serem fatores determinantes da força muscular e amplitude de movimento nas fases da remada ${ }^{12,13}$. Enfim, um perfil favorável pode ser considerado como um fator importante para o desenvolvimento do remador em adição a técnica e experiência. Esse perfil identificado, pode ser utilizado para identificação de talentos e desenvolver avaliações específicas. Atualmente, os estudos analisam as variáveis que predizem o desempenho nos 2000m em remoergômetro, porque essa é a distância oficial no remo olímpico (RO). Pelo contrário, no RE ainda não foi verificado qual o modelo de predição pode predizer o desempenho nos 500m em remoergômetro nos remadores em escaler. Este artigo tem como objetivo demonstrar a aplicação de diferentes modelos de regressão para predizer o desempenho no teste máximo de 500m em remoergômetro com o uso das variáveis antropométricas, composição corporal, consumo de oxigênio pico e força dos remadores em escaler da Escola Naval.

\section{MATERIAIS E MÉTODOS}

Participaram 20 remadores (21,35 $\pm 0,98$ anos) da Escola Naval, representes da equipe de remo em escaler. Todos assinaram um Termo de Consentimento Livre e Esclarecido. A realização da pesquisa recebeu aprovação institucional quanto aos seus aspetos éticos (Parecer 012/14). Foram incluídos na amostra os atletas com um mínimo de três anos de prática na modalidade.

Para o teste máximo de 500 metros no remoergômetro, o de força máxima e a avaliação antropométrica, os remadores foram distribuídos em dois grupos. Cada remador compareceu duas vezes no laboratório da Escola Naval para o teste máximo de 500 metros no remoergômetro; no primeiro dia foram realizados 10 testes em 10 atletas e no segundo dia consecutivo mais 10 testes nos restantes e após 72 horas o reteste com mais dois dias consecutivos. Todos os remadores com um intervalo de 48 horas realizaram os testes de força máxima na remada deitada, no agachamento e no levantamento terra, cada remador concluiu o teste em seis dias consecutivos.

Para testar a confiabilidade dos dados, após 72 horas foram retestados em mais seis dias consecutivos ${ }^{14}$. A avaliação da composição corporal e dados biométricos foi realizado no Hospital Naval Marcílio Dias (HNMD) com o exame de absortometria de raio-X de dupla energia (DEXA). As medidas antropométricas e de força seguiram o proposto por McNeely et al. (2005) ${ }^{14}$.

\section{Teste máximo de $\mathbf{5 0 0}$ metros no remoergômetro}

O teste máximo de $500 \mathrm{~m}$ foi realizado em um remoergômetro Concept 2 modelo D (Concept, Morrivile, VT, USA). O protocolo de Ingham et al. ${ }^{15}$ foi adaptado, sendo reduzido a distância de 2000 metros para 500 metros, a distância da prova do RE, e ainda foi realizado com a resistência máxima do remoergômetro (10). O teste foi antecedido por um aquecimento de 10 min com uma carga auto-selecionada ${ }^{15}$. Para testar a reprodutibilidade dos dados, após 72 horas de recuperação do teste, todos os remadores realizaram o reteste, que seguiu o mesmo protocolo do teste ${ }^{14}$.

\section{Testes de força máxima}

Todos os atletas tinham no mínimo três anos de experiência com o treinamento de força. Após um breve alongamento e aquecimento, a força muscular máxima, através do teste de uma repetição máxima (1-RM), foi determinada por três tentativas de uma repetição, com intervalo de três minutos ${ }^{16}$, nos respectivos exercícios: a remada deitada, agachamento e levantamento terra ${ }^{14}$, utilizando amplitude natural do movimento, com exceção do exercício agachamento, em que se realizou a fase excêntrica até que a coxa do sujeito ficasse paralela ao solo. Um banco foi colocado atrás, para segurança dos atletas e para facilitar o ângulo estabelecido. Foi determinado a carga máxima após a falha concêntrica. A velocidade de movimento foi auto selecionada e não foi permitida paragem do movimento entre a fase concêntrica e excêntrica. Não ocorreu estimulo verbal. Em todos os testes forma usadas barras, discos e anilhas padronizadas da marca Physicus e a execução dos movimentos foi supervisionada por um técnico especialista em treino de força.

\section{Avaliação cardiorrespiratória}

O protocolo do teste incremental utilizado no presente estudo para determinar $\mathrm{VVO}_{2}$ pico dos atletas foi apresentado em alguns estudos ${ }^{9,17}$ e foi adaptado para o RE. Para o teste, os atletas realizaram um aquecimento de 10 minutos livre, no remoergômetro, ajustado com a maior resistência (configuração em 10). Os remadores tiveram que manter de 28 a 30 remadas por minuto. No $1^{\circ}$ estágio a potência foi de 100 watts, sendo que a mesma foi elevada 25 watts de dois em dois minutos em cada estágio. Ao mudar de estágio, o investigador registrou a mudança no analisador de gases e frequência cardíaca (FC). Durante o teste, o investigador questionou os remadores, através de linguagem gestual, se tudo está bem com os mesmos. O término do teste foi ditado pela incapacidade de manter a potência requerida até três remadas.

\section{Avaliação da composição corporal}

Foi utilizado o aparelho de absortometria de raio-X de dupla energia (DEXA) da marca Lunar DPX NT+73983 - GE Healthcare, para avaliar a composição corporal por segmento e total. Para medir a composição corporal com maior confiabilidade se utiliza a absortometria de raio- $X$ de dupla energia (DEXA) ${ }^{18}$, sendo uma técnica de referência ${ }^{19}$. E essa técnica tem conseguido estimar valores da composição corporal de forma segura, com erros menores 20,21 .

\section{Análise estatística}

Foram utilizados procedimentos estatísticos para caracterizar os valores das diferentes variáveis em termos de tendência central e dispersão. Na análise inferencial foi testada a normalidade e homocedasticidade das variáveis através do teste Teste de Kolmogorov-Smirnov e do teste de Levene. Foi realizado ajuste de um modelo de regressão linear simples com cada variável preditora. No modelo de análise de regressão múltipla com todas as variáveis preditoras que apresentaram significância estatística e coeficiente de determinação foi aplicado o método Stepwise. 
Foram ajustados quatro modelos de regressão múltipla de predição que utilizaram: o resultado da antropometria-potência que combinou as variáveis antropométricas com os watts máximo e médio; o resultado do $\mathrm{VO}_{2}$ Pico que combinou as variáveis antropométricas com o VO 2 Pico; o resultado da força-potência que combinou as variáveis dos testes de 1RM com os watts máximo e médio e o resultado de todos os resultados que combinação todas as variáveis. A confiabilidade do modelo de regressão foi expressa pelo coeficiente de determinação (R2) e o (SEE). 0 coeficiente de determinação ajustado, foi utilizado para avaliar a proporção da variação que poderia ser explicada pela variável independente. De acordo com a literatura 22 , o coeficiente de determinação ajustado, considera ambos tanto o número de variáveis preditoras quanto o tamanho da amostra. Para determinar a relação da performance com todos os parâmetros medidos foi utilizado a correlação de Pearson. 0 nível de significância foi fixando em $p<0,05$. Os dados foram analisados com o software estatístico SPSS 16 (Chicago, IL, USA).

\section{RESULTADOS}

Os dados das características motoras gerais e sua correlação com performance nos 500 metros no teste no remoergômetro são apresentados na Tabela 1.

As variáveis estatura, envergadura, 1RM agachamento, massa magra e watt médio tiveram, isoladamente, influência significativa sobre o tempo de percurso dos remadores. Desta forma, foi necessário ajustar um modelo múltiplo com todas estas variáveis de forma que quando aplicado o método Stepwise a única variável constante no modelo foi a potência média $(r=0,918 ; p>0,000)$ e um coeficiente de determinação e um erro padrão da estimativa ( $\mathrm{R} 2=0,843 ; \mathrm{SEE}=0,08$ ) que consegue explicar 84,3\% da variação da velocidade média. Assim, a equação que permite estimar a velocidade média será: $500 \mathrm{~m}(\mathrm{~m} / \mathrm{s})=3,467+0,004^{*}$ (watt médio). Algumas equações opcionais consideraram a massa magra ( $p=0,021)$ que consegue explicar $25,8 \%$ da variação dos dados ( $R 2=0,258)$ ficando: $500 \mathrm{~m}(\mathrm{~m} / \mathrm{s})=2,783+0,002 *$ (massa magra), a estatura $(p=0,039)$ com variação dos dados $(R 2=0,215)$ que explica 21,5\% com a equação: $500(\mathrm{~m} / \mathrm{s})=2,791+0,015^{*}$ (estatura) e a envergadura $(p=0,039)$ com a variação dos dados $(R 2=0,216)$ que explica $21,6 \%$ e com a equação: $500(\mathrm{~m} / \mathrm{s})=2,788+0,014^{*}$ (envergadura). A velocidade média no remoergômetro apresentou forte correlação com watt médio $(r=0,918)$ e massa magra $(r=0,508)$. As demais foram correlações moderadas com a estatura $(r=0,464)$, envergadura $(r=0,465)$ e 1RM agachamento $(r=0,446)$. Os Resultados do ajuste de um modelo de regressão linear simples para a velocidade média são apresentados na Tabela 2.

Os diferentes modelos de predição da performance no remoergômetro utilizando as varáveis deste estudo são apresentados na Tabela 3.

Tabela 1. Característica motoras gerais e performance nos 500m dos remadores.

\begin{tabular}{|c|c|c|c|c|c|c|}
\hline & Média & \begin{tabular}{|l|} 
Desvio- \\
padrão \\
\end{tabular} & Mínimo & Máximo & $r(500 m)$ & Valor $p$ \\
\hline Idades (anos) & 21,35 & 0,98 & 19,00 & 23,00 & $-0,17$ & 0,46 \\
\hline Estatura $(\mathrm{cm})$ & 174,72 & 6,29 & 165,00 & 189,00 & 0,46 & $0,03^{*}$ \\
\hline Envergadura $(\mathrm{cm})$ & 185,18 & 6,66 & 174,90 & 200,30 & 0,46 & $0,03^{*}$ \\
\hline Massa corporal total (kg) & 79,06 & 6.06 & 69,00 & 89.90 & 0,30 & 0,18 \\
\hline Massa Magra $(\mathrm{kg})$ & 62,00 & 4,22 & 56,77 & 69,40 & 0,50 & $0,02^{*}$ \\
\hline Massa Gorda $(\mathrm{kg})$ & 13,33 & 5,18 & 5,79 & 20,78 & $-0,16$ & 0,47 \\
\hline Percentual de Gordura (\%) & 17,21 & 5,56 & 7,40 & 24,50 & $-0,17$ & 0,45 \\
\hline $\mathrm{Vo}^{2} \mathrm{PiCO}\left(\mathrm{ml} / \mathrm{kg} / \mathrm{min}^{-1}\right)$ & 50,32 & 6,81 & 40,19 & 67,52 & $-0,02$ & 0,92 \\
\hline FC Pico (bpm) & 203,60 & 2,43 & 200,00 & 208,00 & $-0,06$ & 0,84 \\
\hline 1RM levantamento terra $(\mathrm{kg})$ & 135,50 & 25,60 & 102,00 & 182,00 & 0,37 & 0,10 \\
\hline 1RM agachamento $(\mathrm{kg})$ & 127,50 & 19,86 & 98,00 & 158,00 & 0,44 & $0,04^{*}$ \\
\hline 1RM remada deitada $(\mathrm{kg})$ & 86,00 & 5,98 & 72,00 & 92,00 & 0,21 & 0,36 \\
\hline Watt máximo $(\mathrm{w})$ & 697,85 & 103,08 & 542,00 & 896,00 & 0,33 & 0,14 \\
\hline Watt médio (w) & 433,65 & 42,87 & 358,00 & 505,00 & 0,91 & $<0,01^{* *}$ \\
\hline Vel. Remoergômetro (m/s) & 93,20 & 3,47 & 88,00 & 99,00 & 1 & 0,19 \\
\hline
\end{tabular}

Tabela 2. Resultados do ajuste de um modelo de regressão linear simples para a velocidade média no remoergômetro.

\begin{tabular}{|c|c|c|c|c|}
\hline Variáveis & Intercepto & Coeficiente & R2 & Valor $p$ \\
\hline Idades (anos) & 6,125 & $-0,035$ & 0,03 & 0,46 \\
\hline Estatura $(\mathrm{cm})$ & 2,791 & 0,015 & 0,21 & $0,03^{*}$ \\
\hline Envergadura $(\mathrm{cm})$ & 2,788 & 0,014 & 0,21 & $0,03^{*}$ \\
\hline Massa corporal (kg) & 4,571 & 0,010 & 0,09 & 0,18 \\
\hline Massa Magra $(\mathrm{kg})$ & 2,622 & 0,004 & 0,25 & $0,02^{*}$ \\
\hline Massa Gorda $(\mathrm{kg})$ & 5,459 & $-0,007$ & 0,02 & 0,47 \\
\hline Percentual de gordura (\%) & 5,165 & $-0,024$ & 0,03 & 0,45 \\
\hline $\mathrm{VO}_{2} \mathrm{Pico}(\mathrm{ml} / \mathrm{kg} / \mathrm{min})$ & 5,406 & $-0,001$ & $<0,01$ & 0,92 \\
\hline FC Pico (bpm) & 2,500 & 0,002 & $<0,01$ & 0,84 \\
\hline 1RM levantamento terra (kg) & 4,980 & 0,003 & 0,13 & 0,10 \\
\hline 1RM agachamento $(\mathrm{kg})$ & 4,798 & 0,004 & 0,19 & 0,04 \\
\hline 1RM remada deitada $(\mathrm{kg})$ & 4,760 & 0,007 & 0,04 & 0,36 \\
\hline Watt máximo (watt) & 4,914 & 0,001 & 0,11 & 0,14 \\
\hline Watt médio (watt) & 3,467 & 0,004 & 0,84 & $<0,01^{* *}$ \\
\hline Vel. Média remoergômetro & 3,297 & 0,732 & 0,09 & 0,19 \\
\hline
\end{tabular}

${ }^{*} p>0.05,{ }^{* *} p>0.01$

Tabela 3. Equações de regressão múltipla, R2, variação e SEE para diferentes modelos.

\begin{tabular}{|c|c|c|}
\hline Modelos & Equações de regressão múltipla & $\begin{array}{l}\text { R2 } \\
\text { Variação } \\
\text { SEE }\end{array}$ \\
\hline \multirow{3}{*}{$\begin{array}{l}\text { Antropometria- } \\
\text { potência }\end{array}$} & \multirow{3}{*}{$\begin{array}{l}500 \mathrm{~m}(\mathrm{~m} / \mathrm{s})=4,603+\left(-0,002^{*} \text { idade }\right)+\left(-0,001^{*} \text { estatura }\right) \\
+\left(0,006^{*} \text { massa gorda }\right)+\left(0,093^{*} \text { massa corporal }\right) \\
+\left(-0,104^{*} \text { massa magra }\right)+\left(-0,093^{*} \text { percentual de }\right. \\
\text { gordura })+\left(0.000^{*} \text { watt máximo }\right)+\left(0,004^{*} \text { watt }\right. \\
\text { médio })+0,06\end{array}$} & 0,92 \\
\hline & & $88 \%$ \\
\hline & & 0,06 \\
\hline \multirow{3}{*}{$\mathrm{VO}_{2}$ Pico } & \multirow{3}{*}{$\begin{array}{l}500 \mathrm{~m}(\mathrm{~m} / \mathrm{s})=3,137+\left(0,000^{*} \text { massa corporal }\right) \\
+\left(0,011^{*} \text { massa magra }\right)+\left(-0,003^{*} \mathrm{VO}_{2} \text { Pico }\right)+ \\
\left(0,000^{*} \text { watt máximo }+\left(0,004^{*} \text { watt médio }\right)+0,07\right.\end{array}$} & 0,88 \\
\hline & & $84 \%$ \\
\hline & & 0,07 \\
\hline \multirow{3}{*}{ Força - potência } & \multirow{3}{*}{$\begin{array}{l}500 \mathrm{~m}(\mathrm{~m} / \mathrm{s})=5,195+\left(0,117^{*} \text { massa corporal }\right)+ \\
\left(-0,138^{*} \text { massa magra }\right)+\left(0,001^{*} 1 \mathrm{RM} \text { levantamento }\right. \\
\text { terra })+\left(0,002^{*} 1 \mathrm{RM} \text { agachamento }\right)+\left(-0,004^{*} 1 \mathrm{RM}\right. \\
\text { remada deitada })+\left(0,000^{*} \text { watt máximo }\right)+\left(0,004^{*} \text { watt }\right. \\
\text { médio })+\left(-0,114^{*} \text { percentual de gordura }\right)+0,06\end{array}$} & 0,93 \\
\hline & & $89 \%$ \\
\hline & & 0,06 \\
\hline \multirow{3}{*}{$\begin{array}{l}\text { Combinação de } \\
\text { todas variáveis }\end{array}$} & \multirow{3}{*}{$\begin{array}{l}500 \mathrm{~m}(\mathrm{~m} / \mathrm{s})=4,018+\left(-0,005^{*} \text { idade }\right)+\left(0,000^{*} \text { estatura }\right) \\
+\left(0,104^{*} \text { massa corporal }\right)+\left(-0,119^{*} \text { massa magra }\right)+ \\
\left(0,004^{*} \text { massa gorda }\right)+\left(-0,105^{*} \text { percentual de gordura }\right) \\
+\left(-0,004^{*} \mathrm{Vo}^{2} \mathrm{Pico}\right)+\left(0,006^{*} \mathrm{FC} \text { Pico }\right)+\left(0,000^{*} 1 \mathrm{RM}\right. \\
\text { levantamento terra })+\left(0,001^{*} 1 \mathrm{RM} \text { agachamento }\right)+ \\
\left(-0,003^{*} 1 \mathrm{RM} \text { remada deitada }\right)+\left(0,000^{*} \text { watt máximo }\right) \\
+\left(0,004^{*} \text { watt médio }\right)+0,08\end{array}$} & 0,94 \\
\hline & & $82 \%$ \\
\hline & & 0,08 \\
\hline
\end{tabular}

\section{DISCUSSÃO}

Os resultados demonstraram uma forte correlação entre a potência média e a massa magra com a velocidade média no remoergômetro, o que confirma que a massa magra exerce forte influência sobre o desempenho no remoergômetro ${ }^{13,23-26}$, sugere-se, de acordo com os dados apresentados, que uma ênfase no treinamento de força para aumento na massa magra, pode auxiliar na melhora do desempenho nos atletas de remo. Com efeito, Gallagher et al. ${ }^{27}$ avaliaram 18 remadores, e observaram que o treinamento de força duas vezes por semana, durante oito semanas, mostrou uma melhora no desempenho.

O estudo de Izquierdo-Gabarren et al. ${ }^{8}$ avaliaram fatores fisiológicos preditores do desempenho e também encontraram correlação significativa entre massa magra e desempenho no remoergômetro. Em outro estudo' aonde foram avaliados os preditores de desempenho, os autores encontraram forte correlação entre a massa magra $(r=-0,812 ; p<0,01)$, a estatura $(r=-0,801 ; p<0,01)$, o agachamento 1RM $(r=-0,755 ; p<0,01)$, a envergadura $(r=-0,715 ; p<0,01)$ e potência média $(r=-0,796 ; p<0,01)$ com o desempenho no remoergômetro, propondo que essas variáveis podem predizer o desempenho, assim como os dados apontados no presente estudo. 
Bourgois et al..$^{13}$ avaliaram a antropometria de 383 remadores, os resultados determinaram que a estatura, massa magra e envergadura estão diretamente relacionadas com o desempenho, por serem fatores determinantes da força muscular. Visto que, deve existir um sincronismo ideal entre cada fase da remada ${ }^{28}$.

Um estudo ${ }^{8}$ apresentou correlação entre a massa magra $(r=0,53$; $\mathrm{p}<0,05)$ e o teste incremental no remoergômetro além disso, massa magra ( $r=0.58 ; p<0,01)$ com o teste de resistência de 20 minutos no remoergômetro. Em outro estudo, os autores ${ }^{29}$ relataram correlações entre o agachamento $(r=-0,536)$ e a estatura $(r=-0,837)$ com o desempenho nos 2000m em remoergômetro. Continuam os autores, na análise de regressão múltipla utilizando as variáveis agachamento e estatura e verificaram um coeficiente de determinação ( $R 2=0,842 ; p<0,05)$, explicando então 84,2\% da variação do desempenho.

Com isso, técnicos e preparadores físicos utilizam parâmetros antropométricos, para selecionar jovens remadores ${ }^{30}$. No entanto, a utilização exclusiva dos parâmetros antropométricos como preditores do desempenho no remo, para selecionar atletas e formar equipes, pode apresentar limitações. Ainda, embora a técnica na execução da remada e a formação da tripulação devam ser sincrônicas, diferenças no condicionamento aeróbio e anaeróbio também influenciam o desempenho.

Quanto ao efeito dos modelos de predição, o presente estudo observou que a combinação de todas as variáveis pode explicar 94\% da variação do desempenho, a força-potência pode explicar 93\%, a antropometria-potência pode explicar 92\% e o VO 2 Pico pode explicar 88\%, colaborando com os estudos anteriores de Akça ${ }^{1}$ e Jurimae et al. ${ }^{11}$. Também a pesquisa de Lawton et al. ${ }^{31}$ com 19 remadores apresentou o agachamento como preditor do desempenho nos 500m (R2=0,59; SEE=6,3) enquanto outro estudo ${ }^{9}$ buscou modelos de regressão para prever o desempenho de remadores nos $2000 \mathrm{~m}$, verificando-se que a potência média apresentou um coeficiente de determinação de R2=0,75 (SEE=6,37).

Em síntese, a correlação entre a potência média, massa magra, estatura, envergadura e 1RM agachamento com o desempenho do remador em escaler encontradas no presente estudo podem ser explicadas pela curta distância em avaliação (500m no remoergômetro). Portanto, as equações de predição apresentadas neste estudo podem não ter aplicação em provas de distância superior (ex. 1000m ou 2000m).

Como limitação do presente estudo, podemos identificar o fato da amostra ser intencional e incluir remadores da mesma Escola Naval e que seguem o mesmo método de treino. Assim, os resultados podem não ser generalizáveis para outros grupos de remadores.

\section{CONCLUSÃO}

Em conclusão, as equações antropometria-potência, de força-potência, $\mathrm{VO}_{2}$ Pico no presente estudo, todas combinadas, foram capazes de predizer o desempenho nos 500m em remoergômetro com uma precisão elevada (94\%). Portanto, os valores preditivos das variáveis apresentados neste estudo podem ser vistos como um complemento importante para fins de identificação e seleção de talentos.

Em estudos futuros, não apenas os modelos preditores devem ser utilizados em um número maior de remadores, como também o uso de técnicas de validação cruzada se faz necessário.

Todos os autores declararam não haver qualquer potencial conflito de interesses referente a este artigo.

CONTRIBUIÇÕES DOS AUTORES: Cada autor contribuiu individual e significativamente para o desenvolvimento do manuscrito. FBMS (0000-0002-0468-4644)*, VMR (0000-0002-4996-1414) e JPRGMB (0000-0003-4357-4269) foram os principais contribuintes na redação do manuscrito. FBMS acompanhou todos os atletas nos exames de absortometria de raio-X de dupla energia no Hospital Naval Marcílio Dias. FBMS realizou todas as avaliações da capacidade de força, consumo de oxigênio pico e antropometria. FBMS e VMR, avaliaram os dados da análise estatística. FBMS, JPRGMB e VMR realizaram a pesquisa bibliográfica, a revisão do manuscrito e contribuíram com o conceito intelectual do estudo. ${ }^{*}$ ORCID (Open Researcher and Contributor ID).

\section{REFERÊNCIAS}

1. Akça F. Prediction of rowing ergometer performance from functional anaerobic power, strength and anthropometric components. J Hum Kinet. 2014;41(1):133-42

2. Smith TB, Hopkins WG. Measures of rowing performance. Sports Med. 2012;42(4):343-58.

3. Smith TB, Hopkins WG. Variability and predictability of finals times of elite rowers. Med Sci Sports Exerc. 2011;43(11):2155-60

4. Mäestu J, Jürimäe J, Jürimäe T. Monitoring of performance and training in rowing. Sports Med. 2005;35(7):597-617.

5. Bourdin M, Messonnier L, Hager JP, Lacour JR. Peak power output predicts rowing ergometer performance in elite male rowers. Int J Sports Med. 2004;25(5):368-73.

6. Cosgrove MJ,Wilson J, Watt D, Grant SF. The relationship between selected physiological variables of rowers and rowing performance as determined by a $2000 \mathrm{~m}$ ergometer test. J Sports Sci. 1999;17(11):845-52.

7. Womack CJ, Davis SE, Wood CM, Sauer K, Alavarez J, Weltman A, et al. Effects of training on physiological correlates of rowing ergometry performance. J Strength Cond Res. 1996;10(4):234-8.

8. Izquierdo-Gabarren M, Expósito RG, De Villarreal ES, Izquierdo M. Physiological factors to predict on traditional rowing performance. Eur J Appl Physiol. 2010;108(1):83-92

9. Riechman SE, Zoeller RF, Balasekaran G, Goss FL, Robertson RJ. Prediction of $2000 \mathrm{~m}$ indoor rowing performance using a 30 s sprint and maximal oxygen uptake. J Sports Sci. 2002;20(9):681-7.

10. Coen B, Urhausen A, Kindermann W. Sport specific performance diagnosis in rowing: an incremental graded exercise test in coxless pairs. Int J Sports Med. 2003;24(6):428-32.

11. Jurimae J, Maestu J, Jurimae T, Phil E. Prediction of rowing performance on single sculls from metabolic and anthropometric variables. J Hum Mov Stud. 2000;38(3):123-36

12. Secher $\mathrm{NH}$, Vaage $\mathrm{O}$. Rowing performance, a mathematical model based on analysis of body dimensions as exemplified by body weight. Eur J Appl Physiol Occup Physiol. 1983;52(1):88-93.

13. Bourgois J, Claessens AL, Vrijens J, Philippaerts R, Van Renterghem B, Thomis M, et al. Anthropometric characteristics of elite male junior rowers. Br J Sports Med. 2000;34(3):213-6.

14. McNeely E, Sandler D, Bamel S. Strength and power goals for competitive rowers. Strength Cond J. 2005;27(3):10-5.

15. Ingham SA, Whyte GP, Jones K, Nevill AM. Determinants of 2,000 m rowing ergometer performance in elite rowers. Eur J Appl Physiol. 2002;88(3):243-6

16. Kraemer WJ, Ratamess NA, Fry AC, French D. Strength testing: development and evaluation of methodology. In: Maud PJ, Foster C, editors. Physiological Assessment of Human Fitness. Champaign, IL: Human Kinetics; 1995. p.115-38.
17. Howley ET, Bassett DR Jr, Welch HG. Criteria for maximal oxygen uptake: review and commentary. Med Sci Sports Exerc. 1995;27(9):1292-301

18. Houtkooper LB, Going SB, Sproul J, Blew RM, Lohman TG. Comparison of methods for assessing body-composition changes over 1 y in postmenopausal women. Am J Clin Nutr. 2000;72(2):401-6.

19. Genton L, Hans D, Kyle UG, Pichard C. Dual-energy X-ray absorptiometry and body composition: differences between devices and comparison with reference methods. Nutrition. 2002;18(1):66-70.

20. Malina RM. Quantification of fat, muscle and bone in man. Clin Orthop Relat Res. 1969;65:9-38.

21. Brozek J, Grande F, Anderson JT, Keys A. Densitometric analysis of body composition: revision of some quantitative assumptions. Ann NY Acad Sci. 1963;110:113-40

22. Russell AP, Le Rossignol PF, Sparrow WA. Prediction of elite schoolboy $2000 \mathrm{~m}$ rowing ergometer performance from metabolic, anthropometric and strength variables. J Sports Sci. 1998;16(8):749-54.

23. Yoshiga CC, Higuchi M. Rowing performance of female and male rowers. Scand J Med Sci Sports. 2003;13(5):317-21.

24. Kerr DA, Ross WD, Norton K, Hume P, Kagawa M, Ackland TR. Olympic lightweight and open-class rowers possess distinctive physical and proportionality characteristics. J Sports Sci. 2007;25(1):43-53.

25. Bourgois J, Claessens AL, Janssens M, Van Renterghem B, Loos R, Thomis M, et al. Anthropometric characteristics of elite female junior rowers. J Sports Sci. 2001;19(3):195-202.

26. Mikulic P. Anthropometric and metabolic determinants of 6,000-m rowing ergometer performance in internationally competitive rowers. J Strength Cond Res. 2009;23(6):1851-7.

27. Gallagher D, Dipietro L, Visek AJ, Bancheri JM, Miller TA. The effects of concurrent endurance and resistance training on 2,000-m rowing ergometer times in collegiate male rowers. J Strength Cond Res. 2010;24(5):1208-14.

28. Nevill AM, Beech C, Holder RL, Wyon M. Scaling concept ii rowing ergometer performance for differences in body mass to better reflect rowing in water. Scand J Med Sci Sports. 2010;20(1):122-7.

29. Huang CJ, Nesser TW, Edwards JE. Strength and power determinants of rowing performance. J Exerc Physiol Online. 2007;10(4):43-50.

30. Piotrowski J, Sklad M, Krawczyk B, Majle B. Somatic indices of junior rowers as related to their athletic experience. Biol Sport. 1992;9(3):117-25.

31. Lawton TW, Cronin JB, McGuigan MR. Strength, power, and muscular endurance exercise and elite rowing ergometer performance. J Strength Cond Res. 2013;27(7):1928-35. 